\title{
On part-to-part feedback optimal control in deep drawing
}

\author{
Pascal Fischer ${ }^{\mathrm{a}, *}$, Jörg Heingärtner ${ }^{\mathrm{b}}$, Stephen Duncan ${ }^{\mathrm{c}}$, Pavel Hora ${ }^{\mathrm{a}}$ \\ ${ }^{a}$ Institute of Virtual Manufacturing, ETH Zurich, Technoparkstrasse 1, Zurich, Switzerland \\ ${ }^{b}$ inspire-ivp, Technoparkstrasse 1, Zurich, Switzerland \\ ${ }^{c}$ Department of Engineering Science, University of Oxford, Oxford, United Kingdom
}

\begin{abstract}
Increased complexity in deep drawn parts can result in the manufacturing process becoming more sensitive. The higher sensitivity results in smaller process windows, which means that current process inputs such as material behaviour, temperature and its associated friction changes, significantly influence the quality of the part. For this reason, control algorithms are needed to automatically adjust the machine settings in order to keep the processes inside these small operating windows. This paper uses optimisation based feedback control algorithms to fulfil this task and it is shown that the resulting controller inherently includes integral action. By considering the location of the closed loop poles of the feedback system, the conditions for a stable control behaviour are derived. The performance of the algorithms are illustrated by applying them to simulations of a deep drawing process and a stamping press.
\end{abstract}

Keywords: Deep drawing, feedback control, robustness, optimization

\section{Introduction}

The complexity of deep drawn parts is continually increasing, partly in response to the requirement for more sophisticated designs, but also because improved accuracy of simulations during the design process allows more complex parts to be manufactured. However, this increasing complexity has the effect of reducing the operating window that will allow parts to be made reliably and accurately. The influences that affect the process can be split into two main categories. The first category includes external variations, such as changes in the material properties or the blank thickness, and changes in the dimensions of the cut blank. A detailed study of the effects of these fluctuations on the production of kitchen sinks was carried out by Harsch [1], which highlighted discrepancies between the properties of material from different suppliers. The second category includes time dependant effects and this group can be split into long-term effects like tool wear, and short time effects, such as variations in the temperature increase caused by the plastic work during the forming process. This temperature increase influences the process significantly, as the properties of the lubricants used in deep drawing are highly temperature dependant, which leads to increased friction as observed by Grübler [2] and higher friction results in higher restraining forces, which have to be compensated by a reduction in the blank holder forces, as described by Hortig [3]. The adjustment of the blank holder force can be carried out by an automatic feedback controller that uses a measurement of the temperature, although in practice, it is usually carried out manually and relies on the experience of the machine operator.

\footnotetext{
${ }^{*}$ Corresponding author

Email address: fischer@ivp.mavt.ethz.ch (Pascal Fischer)
} 
In order to achieve a reproducible and automated adjustment of the process, a number of authors have suggested implementing feedback control systems. These controllers can be divided into two classes. The first class changes the settings of the process during the stroke, while the second class adjusts the process on a part-to-part base. Changes during the stroke have been proposed by Siegert [4], where a combination of draw-in and wrinkle height measurement were used as the state variables, while Griesbach [5] proposed a design based on the draw-in. Further control systems based on different choices of state variables have been proposed. Lo and Yang [6] describe one example that uses the combination of the gap between blank holder and die, which is measured with a photoelectric sensor, as state variables for a decision based control algorithm. A different approach is proposed by Hsu et al. [7], which use the punch force as reference trajectory. Due to the limitations in the response time of the actuators for current press systems, Endelt [8] and Barthau [9] proposed systems based on special blank holder designs.

The concept of part-to-part control aims to automate the adjustments made by the machine operators and assumes that the process itself is stable. A number of studies have been conducted about the stability, one example based on measurement data is the work done by Mork [10], which shows that given the appropriate knowledge of the state of the system, which in this case are the material properties and process settings, then this leads to a good prediction of the process outcome. That suggests that a part-to-part control is viable. The approach taken in this paper is to consider the design of a feedback controller as an optimal control problem and it is shown that this results in the same form of controller as the iterative learning control (ILC) approach that has been introduced by Bäume [11] and Endelt [12]. The benefit of considering the controller in terms of optimal control is that it means that the closed loop poles of the feedback loop can be identified, which allows the effect of model uncertainties on the stability of the system to be evaluated. In addition, it shows that integral action is included inherently within these controllers, which explians why the effect of disturbances can be reduced. An extension to the optimal controller that uses a quadratic programming based approach is described, which allows constraints, such as limitations on the blank holder forces, to be incorporated into the calculation of the optimal settings. The paper describes the results of implementing the proposed algorithms in a virtual environment and presents two methods for the identification of the parameters required by the algorithms.

\section{Statement of Control Problem}

Part-to-part feedback control is based on the assumption that the process is repeatable, so that the uncertainties inherent within the machine result in much lower process variations compared to the variations introduced by external influences, such as variations in material properties or the effects of temperature changes. Part to part feedback control requires an estimate of quality of the part, which can either be observed directly via a measurement system that is able to measure the part quality, or indirectly by measuring a representative quantity of the part. In deep drawing, one of the most accessible quantities is the draw-in as has been shown by Griesbach [5]. The relationship between the draw-in and part quality has recently been considered by Harsch [13], who models the part quality by using the draw-in as an input, indicating that the draw-in can be used as a possible variable for representing the state of a deep drawn part. Griesbach's results [5] indicate that the assumption that the process is reproducible is valid.

Figure 1 shows the structure of the control scheme, which consists of a feed-forward compensator and a feedback loop. In the simplest case, the feed-forward control does not make use of measurements and therefore is a form of open-loop control, which applies a pre-determined schedule for the machine settings, as described in Fischer [14]. This paper focuses on the design of a feedback control loop, which is operated in conjunction with the feed-forward compensator. In closed-loop control, a measurement of part quality is 


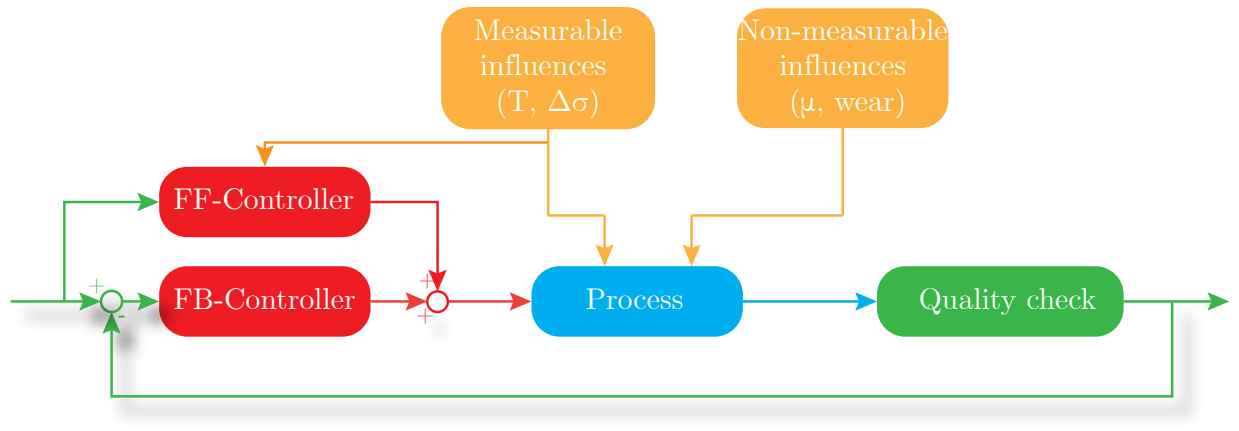

Figure 1: Classical control scheme

obtained from a sensor (or sensors), compared with a reference value and the controller makes adjustments to the machine settings to compensate for the difference.

In practice, most processes have multiple measurements and multiple actuators. For example, a typical press for deep drawn parts in automotive industry has usually at least four different blank holders. However, the number of measurements is often larger than the number of the actuators and in general, it is not possible to keep all measurement values exactly at their reference values. As a result, the control system minimises a cost function that is a combination of the errors at each sensor. The cost function expresses the difference between a reference draw-in for good part quality and the measured draw-in for each part. The control algorithm determines the settings of the actuators for each part that minimise

$$
\text { minimize }\left\|y_{\text {ref }}-y_{k}\right\|_{2}^{2}
$$

where $y_{k}$ is a vector containing the draw-in that is measured at each of the sensors, with $k=1,2, \ldots$ denoting the part number, and $y_{\text {ref }}$ is the vector of the reference draw-in at each measurement location.

The control input that is applied to the actuators is the sum of $u_{\mathrm{ff}}$, the control action determined by the feed-forward control, plus $u_{k}$, the control input from the feedback controller that is applied to the $k$ th part. The measured output for the $k$ th part can be expressed in the form

$$
y_{k}=f\left(u_{\mathrm{ff}}+u_{k}\right)+d
$$

where $f($.$) denotes the effect of the actuator inputs on the draw-in at each measurement location and d$ is the disturbance that affects the each part. This assumes that there are no dynamic effects in the process, although if dynamics do exist, such as thermal effects, the analysis can be extended to include these. The effect of changes to the inputs applied to the actuators on the draw-in is a nonlinear process, but by expanding (2) in terms of a Taylor series around the input from the feed-forward control and keeping the first two terms, then

$$
y_{k}=f\left(u_{\mathrm{ff}}\right)+J u_{k}+d
$$

where $d$ is the fixed disturbance and $J$ is the Jacobian of $f(u)$ evaluated at $u=u_{\mathrm{ff}}$, so that the $i, j$ th element 
of the Jacobian is given by

$$
[J]_{i, j}=\left.\frac{\partial f_{i}}{\partial u_{j}}\right|_{u_{\mathrm{ff}}}
$$

The purpose of the feedback controller is to use $y_{k}$, the measurements of the draw-in for the $k$ th part to determine $u_{k+1}$ the actuator inputs for the next part, in order to minimise the difference between $y_{\text {ref }}$ and $y_{k+1}$. Given that

$$
y_{k+1}=f\left(u_{\mathrm{ff}}\right)+J u_{k+1}+d
$$

then subtracting (5) from (3) leads to

$$
y_{k+1}=y_{k}+J \Delta u_{k}
$$

where $\Delta u_{k}=u_{k+1}-u_{k}$. If the error between the reference draw-in and the measured draw-in is denoted by

$$
e_{k}=y_{\text {ref }}-y_{k}
$$

then

$$
e_{k+1}=e_{k}-J \Delta u_{k}
$$

Given $e_{k}$, the error between the reference value and the measurements of the draw-in for the $k$ th part, the aim of the feedback controller is to determine the changes to the actuator inputs that will minimise $e_{k+1}$, the draw-in error for the next part by solving the optimisation problem

$$
\min _{\Delta u_{k}}\left\|e_{k+1}\right\|_{2}^{2}=\min _{\Delta u_{k}}\left\|e_{k}-J \Delta u_{k}\right\|_{2}^{2}
$$

As will be shown below, in practice, this can lead to large changes in the control inputs, particularly when $J$ is ill-conditioned. For this reason, a regularisation term can be included so that the optimisation problem becomes

$$
\min _{\Delta u_{k}}\left\|e_{k}-J \Delta u_{k}\right\|_{2}^{2}+\mu\left\|\Delta u_{k}\right\|_{2}^{2}
$$

where $\mu$ is the regularisation parameter. Including regularisation has the effect of making the feedback control robust to uncertainties in the Jacobian matrix. If there are constraints on the allowable values of the control inputs, these can be incorporated by solving a constrained optimisation problem.

\section{Measurement systems}

For part-to-part process control, the draw-in of every part has to be measured and a number of authors have proposed and tested measurement systems. The systems can be split in in-tool and out of tool based systems.

A widely used system is the tool based measurement of the draw-in that uses laser triangulation as described in Griesbach [5]. The advantage of laser triangulation is that it has the potential to measure the progression of the draw-in during the stroke with high accuracy. One disadvantage of this sensor type is associated with the use of a laser beam, as the beam is only able to follow the blank edge in areas where the 
tool is flat or slightly curved areas. This effect, in combination with the limited space in the tools, restricts the placement of the sensors. Another disadvantage of tool based sensors is the need to determine the positions of the sensors during the design phase, as a repositioning of the sensor during try-out or production would result in a revision of the tooling, as new sensor positions have to be milled. An alternative to laser triangulation is an induction based system as proposed by Neumann [15], which is placed directly in the tool surface and is therefore able to measure areas of the tool where the curvature is large. However, this system is less accurate when used in non-ferromagnetic blanks.

More recent research focuses on the development of optical measurement systems. These measurements systems are usually placed between two drawing stages, which means that they lose information about the progression of the draw-in. On the other hand, there is more flexibility in the positioning of the measurement points. For example, Kraft [16] is able to measure the whole circumference of the part, while Heingärtner [17] focuses on a set of defined positions.

For the controller itself, the choice of measurement system is a secondary issue provided that the sensor accuracy is sufficiently high, which depends on the desired accuracy for the part. However, it is important to ensure that the sensor values are positioned correctly within the tool. Neuhauser [18] proposed a method based on Principle Component Analysis (PCA) in combination with a strong rank revealing QRdecomposition to determine sensor positions that reflect the part quality. The method yields good results for processes that can be described by linear models.

\section{Optimal control}

\subsection{Unconstrained optimisation}

The solution to the unconstrained minimisation problem given in $(10)$ is

$$
\Delta u_{k}=\left(J^{T} J+\mu I\right)^{-1} J^{T} e_{k}
$$

and since $\Delta u_{k}=u_{k+1}-u_{k}$, then

$$
u_{k+1}=u_{k}+\left(J^{T} J+\mu I\right)^{-1} J^{T} e_{k}
$$

By writing

$$
u_{k+1}=\frac{1}{z-1}\left(J^{T} J+\mu I\right)^{-1} J^{T} e_{k}
$$

where $z^{-1}$ is the delay operator, this shows that the feedback control system inherently includes integral action, which explains why it can eliminate any constant disturbance $d$ that affects the process. If the integrator also includes a gain $K$, as shown in figure 2 , then the control law becomes

$$
u_{k+1}=u_{k}+K\left(J^{T} J+\mu I\right)^{-1} J^{T} e_{k}
$$

which takes the same form as the iterative learning control approach proposed by Endelt [12], apart from two differences. Firstly, Endelt's control scheme is defined in terms of the residual $y_{k}-y_{\text {ref }}$, which is the negative of $e_{k}$, so there is a change of sign on the right hand side of (13). Secondly, his scheme is derived from a Gauss-Newton minimisation that includes a line search, which corresponds to a variable gain $K$ for the integrator. 


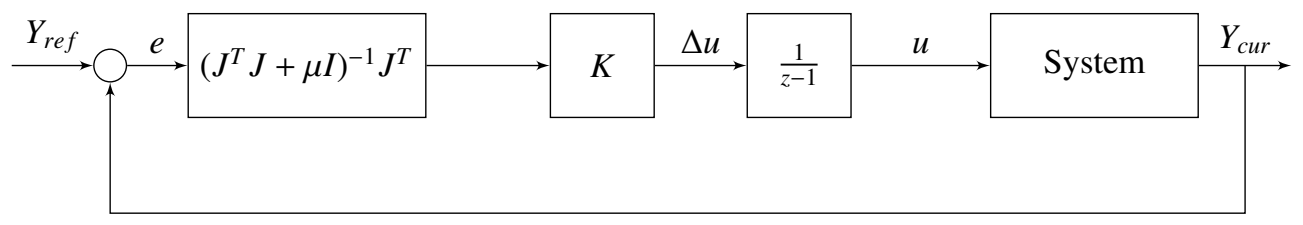

Figure 2: Feedback control for unbounded optimisation

The closed loop stability can be determined by using the singular value decomposition

$$
J=U \Sigma V^{T}
$$

where $\Sigma$ is the diagonal matrix containing the singular values $\left\{\sigma_{1}, \sigma_{2}, \ldots, \sigma_{n}\right\}$, while $U$ and $V$ are orthogonal matrices containing the left and right singular vectors. By applying the same $U$ and $V$ to the controller results in the transformation

$$
\left(J^{T} J+\mu I\right)^{-1} J^{T}=V \Gamma U^{T}
$$

where $\Gamma$ is a diagonal matrix whose $n, n$th element is

$$
[\Gamma]_{n, n}=\frac{\sigma_{i}}{\sigma_{i}^{2}+\mu}
$$

When these transformations are applied, the control scheme takes the form shown in figure 3

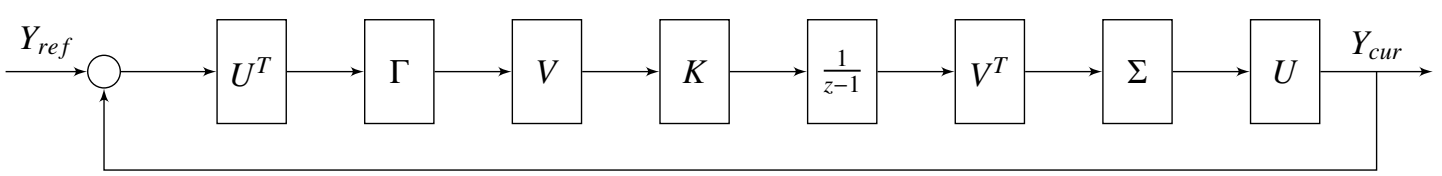

Figure 3: Control scheme for unbounded optimisation with transformations applied

Because $V$ is orthogonal, the system can be decoupled into a set of single-input, single-output (SISO) systems, one for each of the actuators. This decoupling (see figure 4) allows the stability of the overall system to be considered in terms of the closed loop stability of the subsystems. The system is stable if the roots of all closed loop transfer functions of each subsystem $T_{n}(z)$ lie inside the unit circle. The closed loop transfer functions

$$
T_{n}(z)=\frac{\Gamma_{n, n} \sigma_{n} K \frac{1}{z-1}}{1+\Gamma_{n, n} \sigma_{n} K \frac{1}{z-1}}
$$

The fastest transient response is obtained when the gain $K$ is chosen such that

$$
K \Gamma_{n, n} \sigma_{n}=1
$$

in which case, the closed loop system will converge to $y_{\text {ref }}$ in a single step. This can be achieved by choosing $K=1$ and $\mu \rightarrow 0$, so that

$$
\lim _{\mu \rightarrow 0} \Gamma_{n, n} \sigma_{n}=\lim _{\mu \rightarrow 0} \frac{\sigma_{n}}{\sigma_{n}^{2}+\mu} \sigma_{n}=1
$$




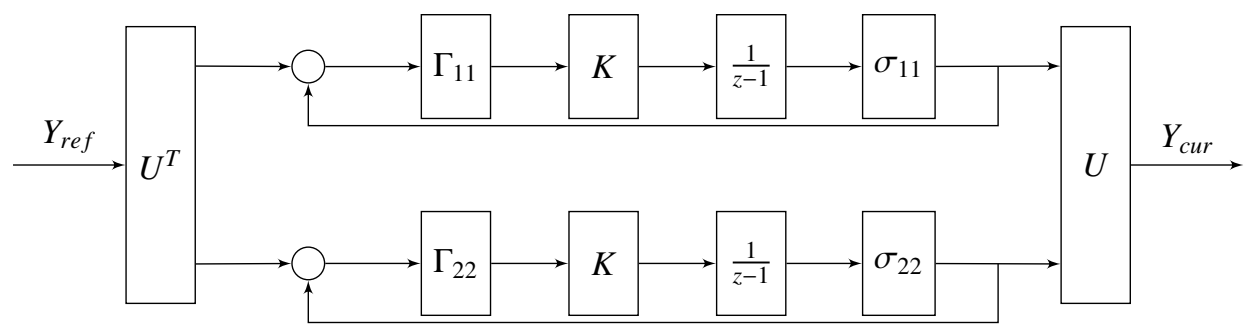

Figure 4: Control scheme when singular value decomposition is applied

Although this gives the fastest possible response, in practice, the value of $\sigma_{n}$ is not known exactly, both due to modelling errors and due to the fact that the control system is based on a Taylor expansion that neglects higher order terms, so the model underlying the controller is not accurate. In particular, for small singular values, the corresponding $\Gamma_{n, n}$ will be large. The overall closed loop system will be stable if each of the individual SISO loops are stable and by calculating the roots of each of the transfer functions, the full system will be stable if a single value of $K$ can be found such that the condition

$$
0<K \Gamma_{n, n} \sigma_{n} \leq 2
$$

is satisfied for each individual loop. When $\Gamma_{n, n}$ is large, any small error in $\sigma_{n}$ can result in this condition being violated. For this reason, it is better to include regularisation by choosing $\mu>0$ in order to limit the magnitude of $\Gamma_{n, n}$. This has the effect of making the feedback control less sensitive to uncertainties in $\sigma_{n}$, at the expense of slowing down the transient response of the loops associated with small values of $\sigma_{n}$, which slows down the overall response of the system due to the coupling of the loops via the $U^{T}$ and $U$ matrices as shown in figure 4

\subsection{Effect of Sensor Positioning}

The feedback control system will particularly sensitive to uncertainties in the singular values when the condition number of the Jacobian, defined as

$$
\kappa=\frac{\sigma_{\max }}{\sigma_{\min }}
$$

is large. The conditioning of $J$ is highly dependant on the sensor positions, so a suitable sensor placement is required. The simplest algorithm for finding the appropriate sensor positions is shown in algorithm 1 . The proposed algorithm is conceptually simple, but in practice, is limited to about twenty sensor positions, as the calculation time rises rapidly for a large number of sensors.

The limitations of algorithm 1 are due to calculating the condition of all possible combinations of $i$ sensors out of $m$ sensors and choosing the combination with the lowest condition. With a rising number of possible sensor positions $m$, the number of combinations rises extremely. Therefore, for higher numbers of possible sensor locations, a basic number of sensor equal to the number of actuators can be found by using strong rank revealing QR factorisations, for example with the algorithm proposed by Gu and Eisenstat [19]. These initial selections can be extended by including redundant sensors for further improvements as shown later. 


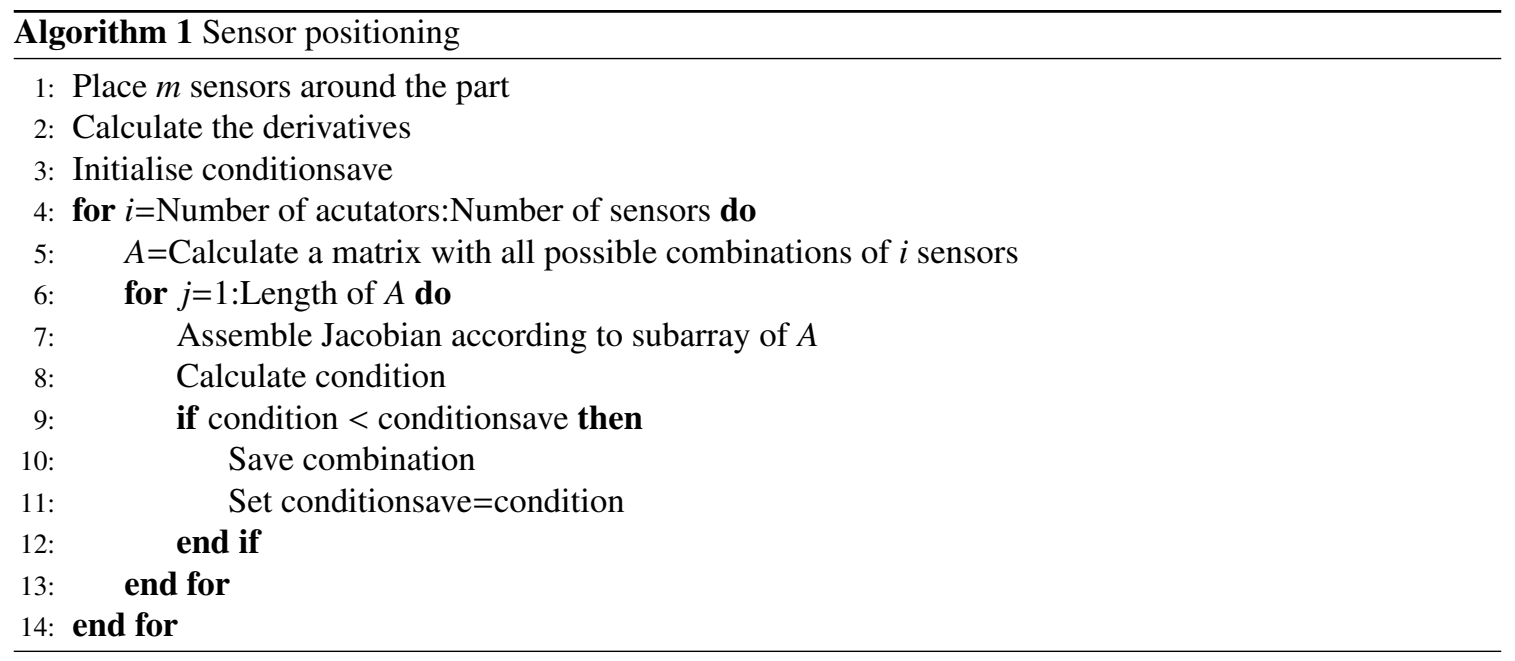

\subsection{Constrained optimisation}

In forming, the process is subject to limits, so a constrained optimisation scheme is introduced. The constraints can arise from machine limitations, such as the maximum force that can be applied to a blank holder cylinder, on to the direct application of quality parameters. In general, machine limitations should not be reached provided that the process has been correctly designed, but in practice constraints do occur. These limitations can be handled by rewriting the control problem as a quadratic programming problem, which minimises a cost function of the special form shown in eq. 23 subject to satisfying equality and inequality constraints.

$$
\begin{gathered}
\min \frac{1}{2} \Delta u^{T} Q \Delta u+e^{T} J \Delta u \\
A_{e q} \cdot u=b_{e q} \\
A \cdot u \leq b
\end{gathered}
$$

where $\Delta u$ is the change in the actuators and

$$
Q=J^{T} J
$$

Provided that $J$ is non-singular, $Q$ will be positive definite and the minimisation problem is convex. The solution of the quadratic problem can be determined using standard QP solvers, for example quadprog in Matlab [20]. The cost function for the constrained problem does not include a regularisation term as the constraints restrict the range of actuator inputs, which has the effect of limiting the magnitude of $\Delta u_{k}$. The quadratic problem has to be solved for every part in a part-to-part control as shown in algorithm 2 . noting that $J$ is the Jacobian at the nominal working point and the solution $\Delta u$ of the quadratic program in the control algorithm. The $A$ can represent machine limitations, which might be the identity matrix, while $b$ might be, the difference between the limit value and the current value (e.g. $b=X_{\text {upper }}-X_{\text {cur }}$ ). The implementation of boundary conditions that reflect quality criteria is more challenging, as usually the models are not linear, therefore, an approximation about the allowable change has to be calculated, either by linear approximations or by applying optimisation techniques. Also, the application of quality criteria is only useful if other parameters, such as temperature and material properties, are known as the estimation of 


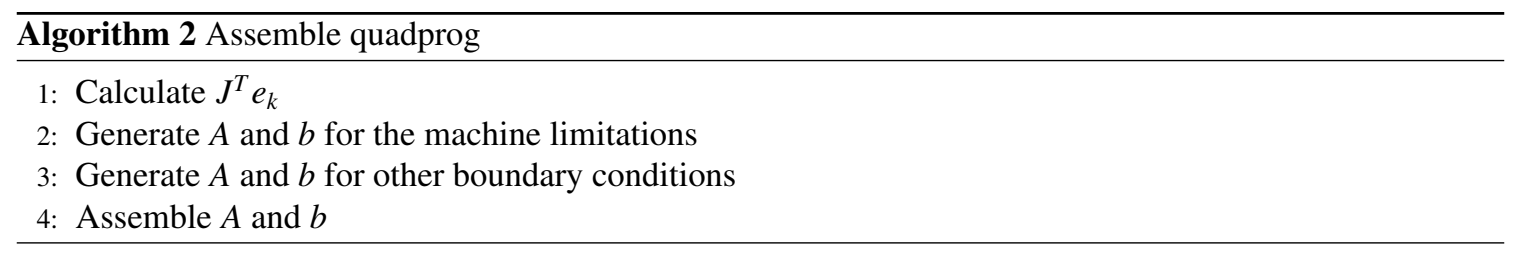

the distance between the working point and the failure limit is highly improved by these parameters. With these additional boundary conditions definable, the quadratic programming problem is suited to use the full knowledge of FEA based meta-models of the process.

For the sensor placement problem, the quadratic programming approach differs from the unconstrained case, as the fixed relation between a sensor position sensitivity and the error is changed to a combined sensitivity and a weighted sensor error. Therefore, it is beneficial to place the sensors not only with regard to the lowest condition, but also with regard to covering most features of the part. The influence of additional sensors and different sensor positions is discussed in section 6.

\section{Parameter identification}

In order to use the control algorithms, it is necessary to have a reliable estimate of the model parameters and in particular, it is necessary to determine the Jacobian of the process. Therefore, two different identification methods are shown: The first method relies on virtual experiments, while the second approach focuses on the experimental identification.

\subsection{Finite element based identification}

Using an estimation procedure based on a finite element based model has the advantage of not only identifying the Jacobian, but also offers the opportunity of identifying a model for a wider design space, as well as generating models for part quality. The first step in this identification process is the generation of a base model that is able to handle all actuators separately and which leads to a good agreement between the virtual model and a part produced by the press when applying the same machine settings. As a result, not only the material parameters have to be identified but also the blank holder system has to be modelled in order to simulate the finished part when different settings are applied to the blank holders. With this baseline simulation in place, the effect of making relatively small deviations from these settings can be modelled. Bonte [21] proposed different methods for designing experiments that will cover different model types. Usually quadratic models with interaction should result in sufficient approximations and therefore, a spacefilling Latin hypercube design should be suitable. Fitting the draw-in changes induced by the actuators on the response surfaces also allows for either numeric or analytical identification of the Jacobian. The quality criteria can be fitted accordingly and used in the constrained control scheme.

\subsection{Experimental identification}

The advantage of the experimental based approach is that it is not influenced by the unknown accuracy of the finite element simulation and is only influenced by the accuracy of the measurement system and the repeatability of the process. The number of experiments usually has to be kept as small as possible in order to minimise disruption to the production line, so the identification of the Jacobian can be either determined by carrying out a full experiment design, as done by Fischer [22], or by using a reduced design which only varies each actuator on its own. The full design has the advantage of the possibility of determining the 
Jacobian inside the design space, while the reduced version is more cost effective. The disadvantage of the full design is, that the number of experiments is also limited by the size of the blank stack, as a different stack might change the response. To account for the inherent process fluctuations, for every setting multiple parts have to be produced and therefore, the limitation in the number of experiments is the size of the blank stack. However, the reduced approach, which only identifies one Jacobian, can usually be completed within a single stack without any issues, although the stability has to be assumed.

\section{Virtual validation}

The virtual validation of the approach is carried out using two different demonstration parts. The first demonstration part is the kitchen sink described by Fischer [23], while the second part is a front fender which is mentioned in Fischer [22].

\subsection{Kitchen sink}

For demonstrating the behaviour of the control algorithm, the first step in the production of a kitchen sink is remodelled in LS-Dyna to account for the five different blank holder forces as shown in figure 5 . The model has four local acting blank holders $\left(F_{1}-F_{4}\right)$ and one global acting one $\left(F_{0}\right)$. The die (in green), as well as the blank holder (blue and red parts) are modelled as elastic bodies with a purely elastic behaviour so that the local behaviour can be determined. The punch as displacement controlled body is modelled as rigid body. The material for kitchen sink is modelled by the Hänsel [24] model and the yield locus of Barlat [25]

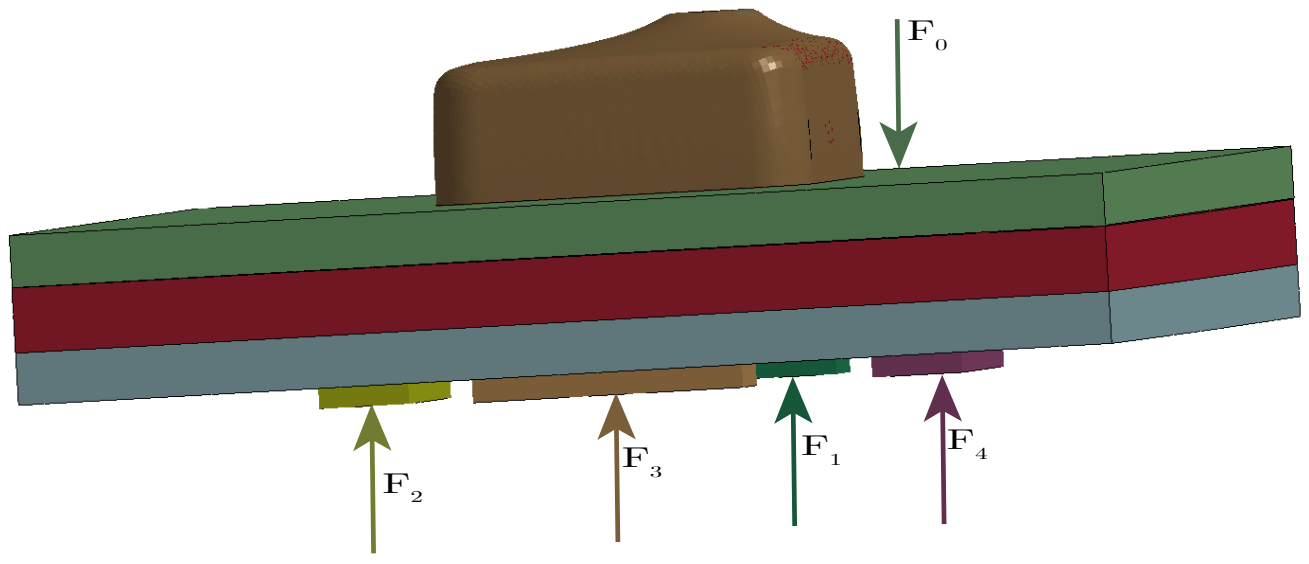

Figure 5: LS-Dyna model

using the data provided by Harsch [1] for Posco stainless steel. The model is solved by the explicit solver for the forming step and the implicit solver for the springback calculation. The draw-in of the base simulation is calibrated by using the production blank holder forces, while the value of the constant friction is adjusted to approximate the draw-in of a measured part.

Based on this model, the blank holder forces and the friction are changed according to the values in table 1 using a Latin hypercube design with 50 runs. The draw-in at the 19 positions is calculated as the displacement of the nodes shown in figure 6 . For the validation runs, the draw-in at these points is modelled by response surfaces with a quadratic function that also includes interactions. 
Table 1: Parameter range for variant simulation

\begin{tabular}{|c|c|c|c|c|c|c|}
\hline Parameter & $F_{0}[\mathrm{kN}]$ & $F_{1}[\mathrm{kN}]$ & $F_{2}[\mathrm{kN}]$ & $F_{3}[\mathrm{kN}]$ & $F_{4}[\mathrm{kN}]$ & $\mu[]$ \\
\hline $\min$ & 1300 & 150 & 150 & 150 & 150 & 0.025 \\
\hline $\max$ & 1700 & 250 & 250 & 250 & 250 & 0.055 \\
\hline nom & 1500 & 200 & 200 & 200 & 200 & 0.035 \\
\hline
\end{tabular}

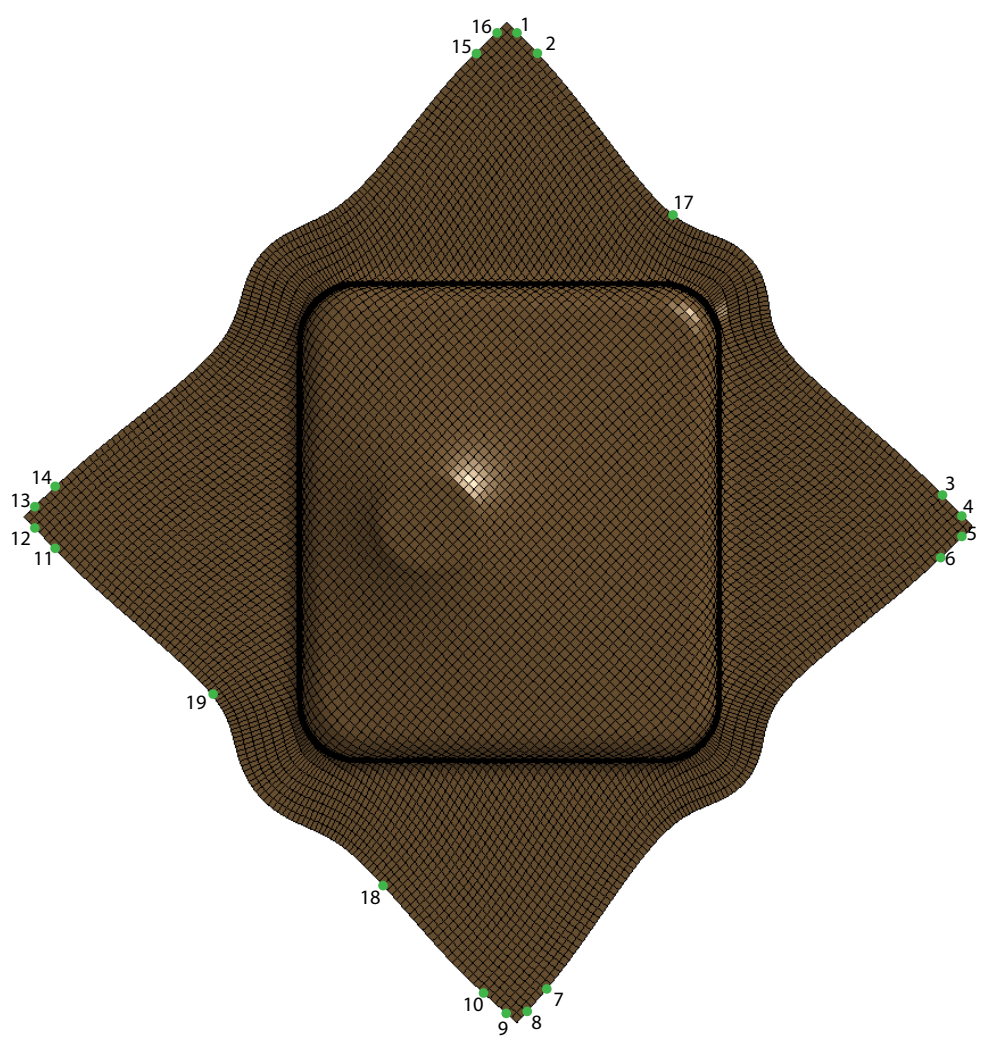

Figure 6: Positions of virtual sensors

The coefficient of regression for all sensors models is higher than 0.9 and therefore this approximation can be used for the virtual test of the proposed control algorithms.

With the models in place, the two control approaches can be tested on their overall behaviour, their reaction on changes in friction, as well as their stability regarding noise.

\section{Unbounded optimisation}

Firstly, suitable positions for the sensors for the control algorithm have to be found. Based on algorithm 1. the best conditioned Jacobian is obtained using positions $6,7,14,16,17$ and 19. In general, a system without noise only needs the same number of sensors as it has actuators, but in the case of the sink, the algorithm identified six locations that gave the best condition number, so a controller with these six sensors and the 
regularisation parameter $\mu=0$ was used as the base design. The base design, as well as the variations, were tested by starting with incorrect process settings, as well as changing the friction from 0.035 to 0.045 after 22 parts. The starting setting for $F_{0}$ is chosen to be higher than the correct value, while the values for the other four blank holders are chosen to be too low. For the nominal control algorithm, the Jacobian at the nominal working point is chosen and the gain $K$ is set to 0.8 . Figure 7a shows the draw-in error of all 19 sensors with the first part being the part with the initialised settings. As expected, the set change in the friction at part 22 results in a high draw-in error. The forces set by the controller (figure 7b) and the draw-in error show that in both cases (incorrect initial conditions and friction change), the controller needs about 4 parts to return to the nominal, except for sensor 18 which continues to have an offset, even with the minimised least square approximation.

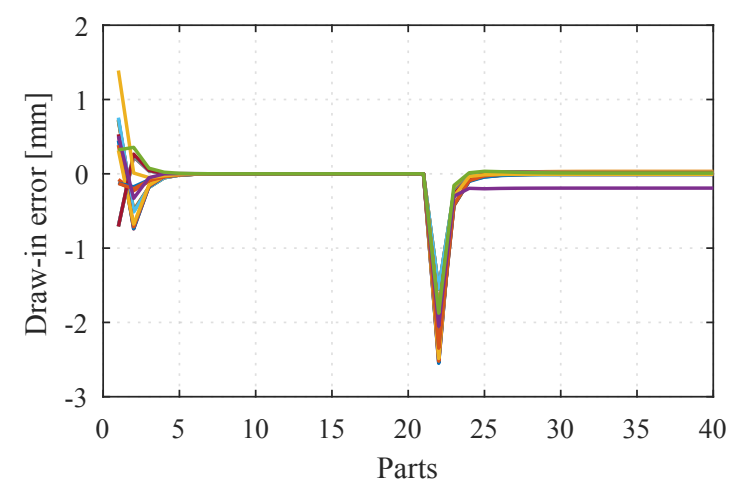

(a) Draw-in reaction

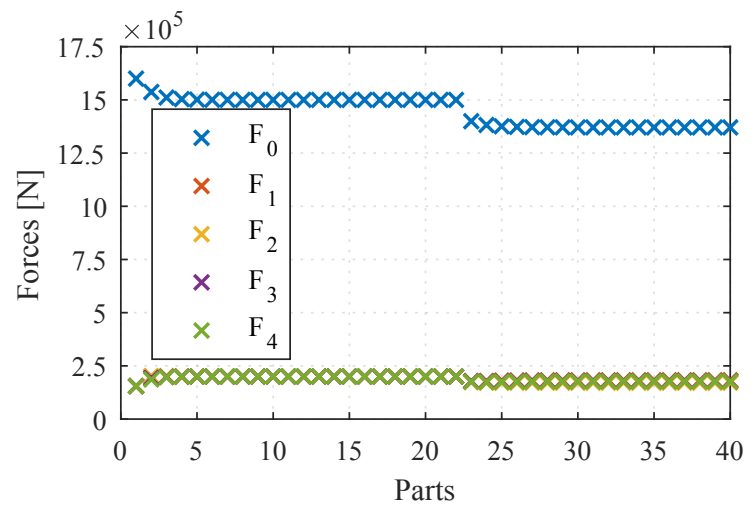

(b) Force reaction

Figure 7: Behaviour of nominal controller

With the nominal controller working, the influence of the different parameters can be checked. Figure 8 shows that a large gain, e.g. $K=1$, can result in significant overshoot in the response compared to the reference, while a small gain, e.g. $K=0.5$, results in slower convergence. In the case of the small $K$, the controller will converge, while using a gain that is too high can result in an unstable behaviour with oscillations, occurring around part 24 in figure $8 \mathrm{~b}$.

By using the approach of minimising the condition of the control matrix, the effect of a badly conditioned controller can be avoided. The effect of a badly conditioned can be observed in figure $9 \mathrm{a}$. This controller has the condition of $\kappa=30$, while the nominal one has the condition $\kappa=11$. The change in conditioning is a result of using sensors 1-3, sensor 4-16 and 18. The controller can be stabilised as shown in figure $9 \mathrm{~b}$ by setting $\mu=2 * 10^{-10}$, which results in a condition $\kappa=3$, but the change in $\mu$ results in a slower response that also loses accuracy, although it is still more accurate compared to an open loop approach that does not use feedback control. Therefore the extension is necessary for the cases where the ideal positions cannot be identified in a virtual model.

In practice, measurement values tend to be subject to variations. To test the behaviour of the control algorithm in the presence of sensor noise, gaussian distributed noise with a standard deviation of $\sigma=0.1$ is added to every sensor value used by the control algorithm. The plots in figure 11 show the true draw-ins without the influence of noise, but when noise is applied to the sensors, using all 19 sensors helps to smooth the controlled result (figure 10b compared to the case with the six sensors. 


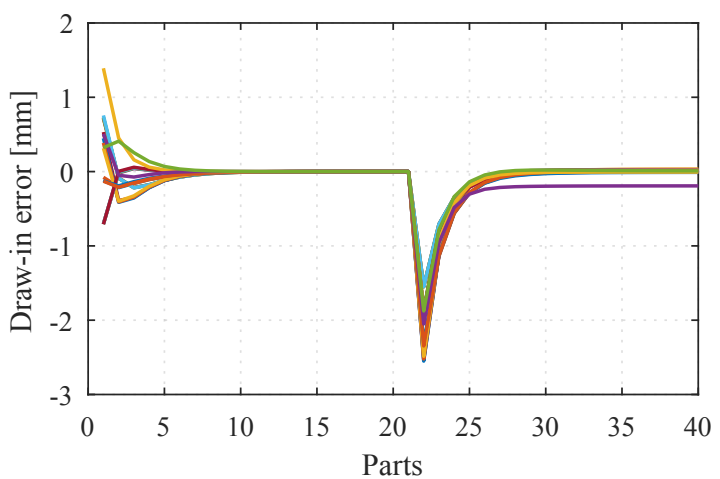

(a) $K=0.5$

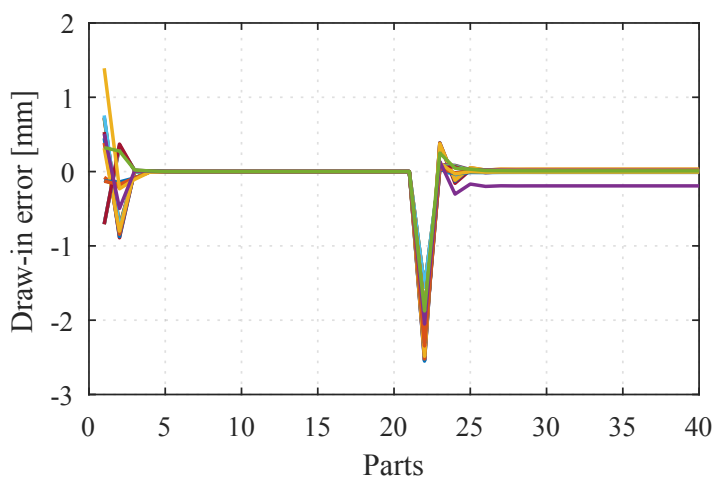

(b) $K=1.0$

Figure 8: Comparison of different gains

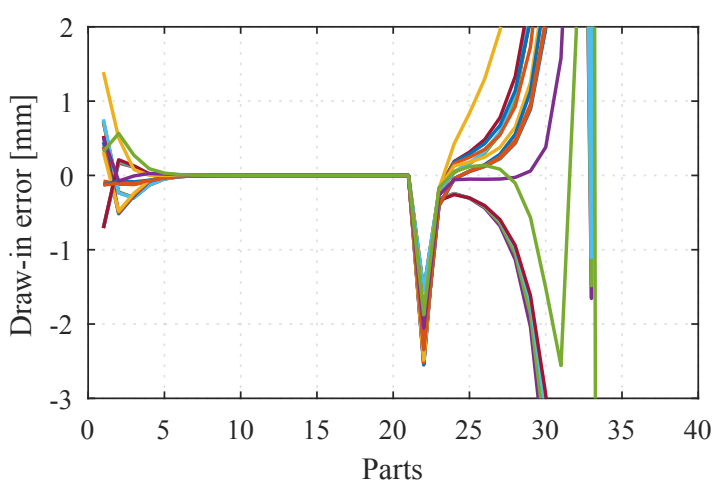

(a) $\mu=0$

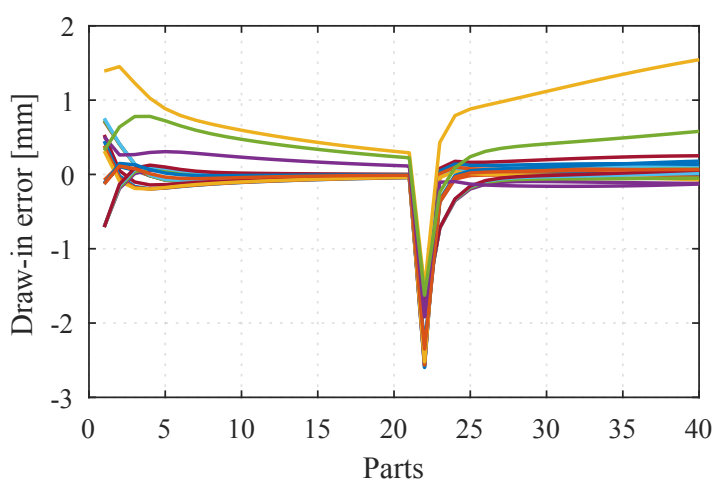

(b) $\mu=2 * 10^{-10}$

Figure 9: Badly conditioned controller

While the reaction on large steps shows the general behaviour of the control algorithm, figure $11 \mathrm{~b}$ shows the reaction on a more practical test scenario. For the test scenario, the same wrong starting point is chosen, but the friction (figure 11a) is modelled with a small variation of $\sigma=0.003$ and is rising between part 10 and 20, to model a temperature rise in the tool. The controlled result shows that the controller is able to compensate the rising friction, but is slightly too aggressive and therefore, the controller overshoots slightly after each major disturbance. Overall, the unconstrained optimisation works well for the virtual model of the kitchen sink. The results also show that the controller will operate satisfactorily in the presence of sensor noise, although the sensors need to be positioned optimally. 


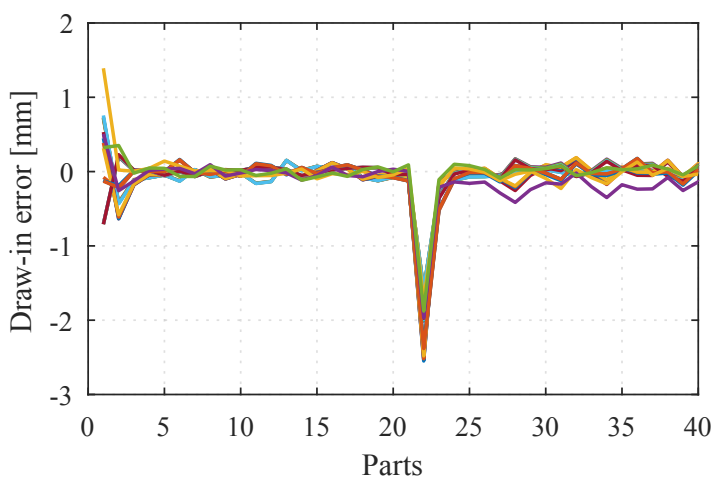

(a) Nominal design with six sensors

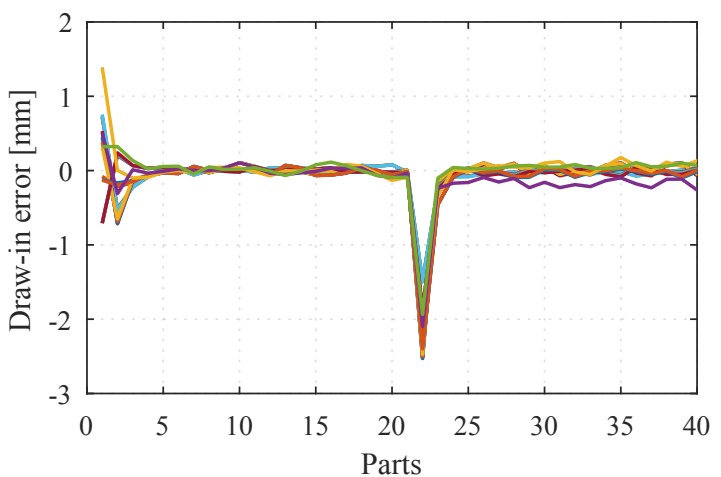

(b) All 19 sensors

Figure 10: Unbounded controller influenced by noise

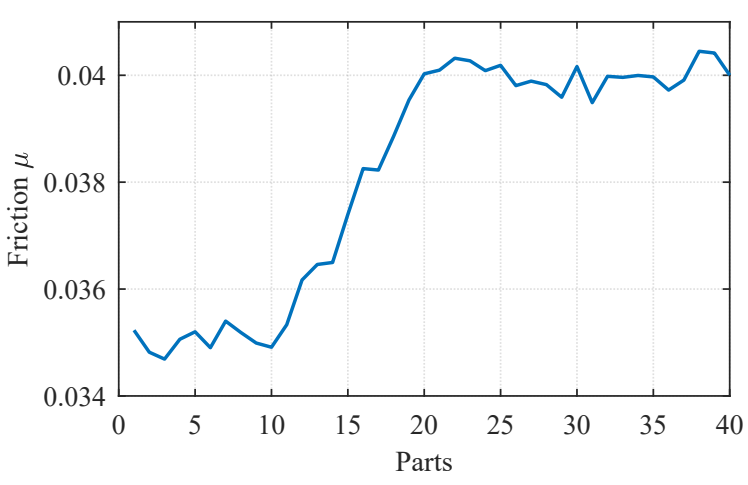

(a) Friction with process noise

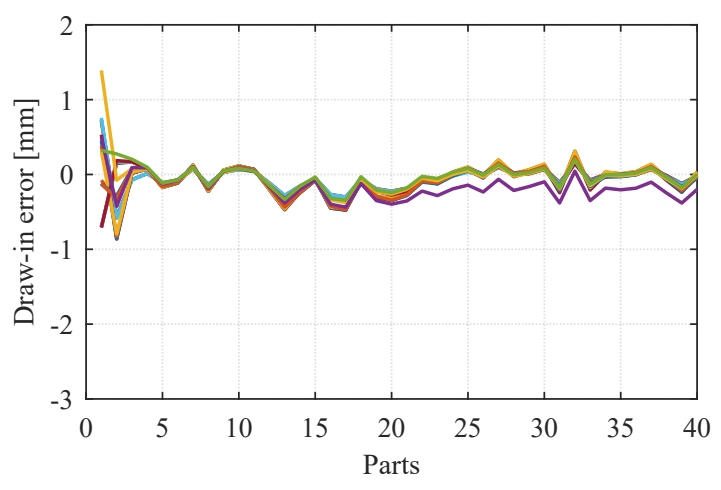

(b) Control response

Figure 11: Unbounded controller and unsteady friction

\section{Constrained optimisation}

For testing the control algorithm in the presence of actuator constraints, the same approach is taken as for the unconstrained controller. Figure 12 shows the results that match the unconstrained case with gain $K=1$.

In the absence of limits on the actuator inputs, the constrained controller has no direct advantage compared to the unconstrained case. The benefit of the quadratic programming based controller arises when bounds are introduced. One possible bound for example would be a minimum allowable force in $F_{0}$, which in this example, is set to $14.5 * 10^{6} \mathrm{~N}$.

The results for the constrained case are given in figure 13, which shows that the controller reacts with a lower than expected force in the blank holders 1-4 to offset the limitation in $F_{0}$. This indicates that the constrained optimisation is beneficial for processes in which the quality limitations are known or for processes which are close to the machine limitations. 


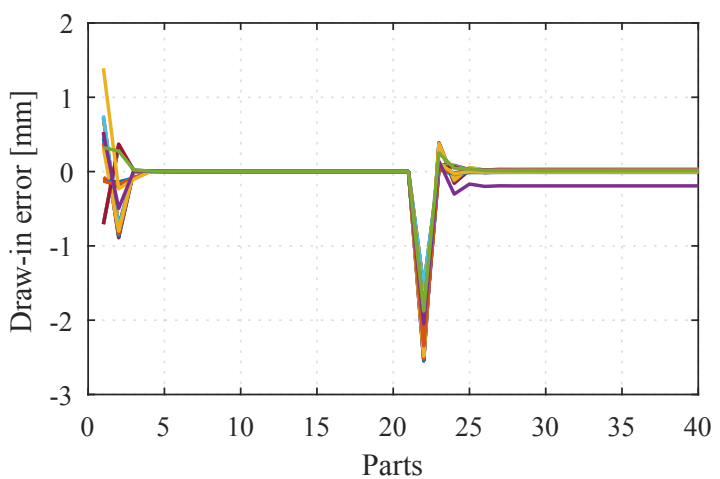

(a) Draw-in reaction

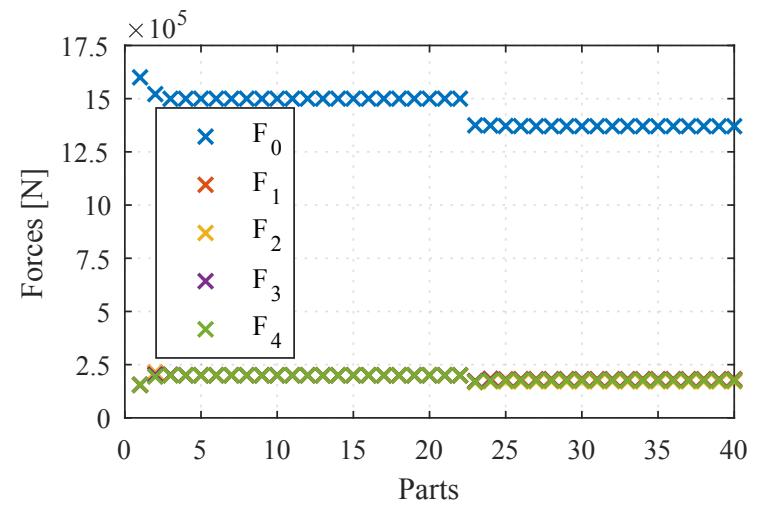

(b) Force reaction

Figure 12: Bounded controller without bounds

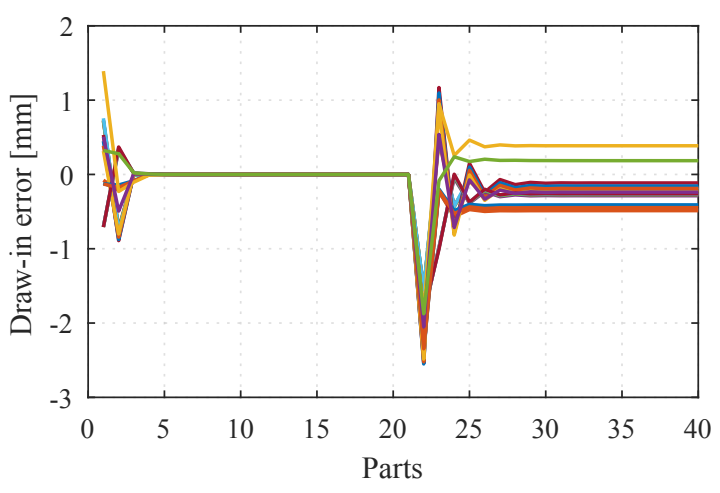

(a) Draw-in reaction

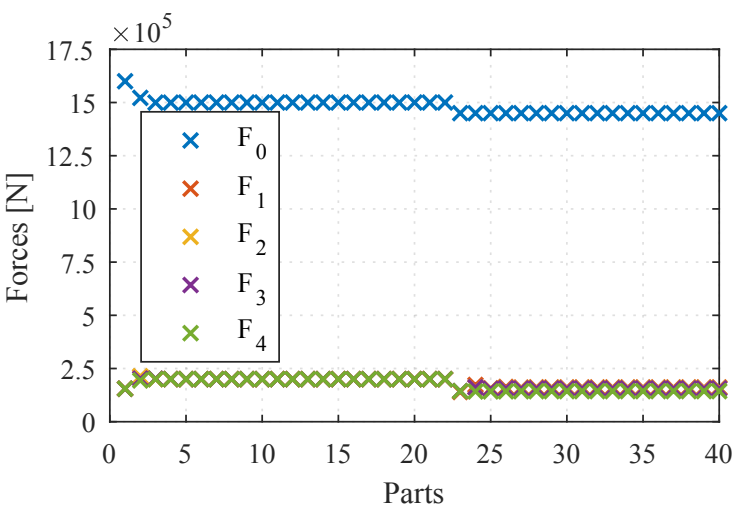

(b) Force reaction

Figure 13: Bounded controller with bounds in $F_{0}$

\subsection{Front fender}

In addition to testing the control approaches on simulation based models, the controller was also tested on the experimentally generated models described by Fischer [22]. The controller uses sensors three and four (figure 14), as they show the best predictions of the draw-in and the controllers based on these two measurements worked well. The six actuators in the figure are reduced to $F_{\text {comb }}$ and $F_{\text {dist }}$, with $F_{\text {comb }}$ being the total accumulated force and $F_{\text {dist }}$ being the difference between each cylinder in the direction of material flow and the corresponding cylinder counter the material flow direction. The used models are based on a nearly full factorial design with nine different settings and 20 parts per setting. The nine data points are than used to fit a model for sensor three and four. The model for sensor three is a quadratic model with 
interaction, while the sensor four is modelled by a quadratic approach.

$$
\begin{aligned}
& F_{\text {comb }}=\sum_{i=1}^{6} B H i \\
& F_{d i s t}=B H 2-B H 1
\end{aligned}
$$

Using this setup, the response of the control algorithms can be evaluated.

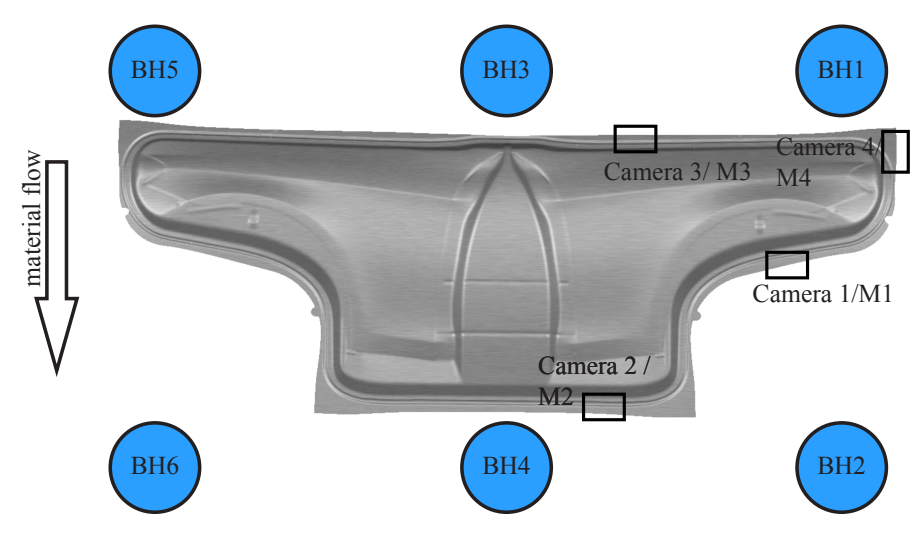

Figure 14: Sensor and actuator positions of the front fender

\section{Unconstrained optimisation}

The front fender with two inputs and two outputs allows for a visualisation of possible Jacobians that allow convergence to the required reference point. In figure 15 all gradients that result in a positive critical gain to converge to the reference $\left(F_{\text {comb }}=2760\right.$ and $\left.F_{\text {dist }}=20\right)$ are green dots, while the negative ones are red dots. From another perspective, choosing the red points as starting points with the Jacobian taken at the reference can result in convergence, but may not converge to the correct reference value.

In figure 16, the convergence using the nominal Jacobian when starting from a red position is shown. The gain is again chosen to be $K=0.8$. The force reaction in figure $16 \mathrm{~b}$ shows that in the first step the difference has a small overshoot, but after four parts, the reference values for the draw-in and the force are achieved.

Starting at a green point in the design space (figure 17) shows the same behaviour with a larger overshoot. Therefore, a reduction of the gain would result in smoother convergence to the reference. As these large overshoots are likely to result in defective parts in a production environment, it is necessary to start with a low gain. However, since the control algorithm converges to the reference in both cases, this indicates that the unconstrained controller works well for the fender.

\section{Constrained optimisation}

The constrained optimisation approach for the fender considered the same cases as the unconstrained, so the green starting point is mainly tested. The results in figure 18 show that the algorithm optimises the cost function in this case, in a similar manner to the unconstrained method, while the difference between both algorithms is mainly the gain in the unbounded case. 


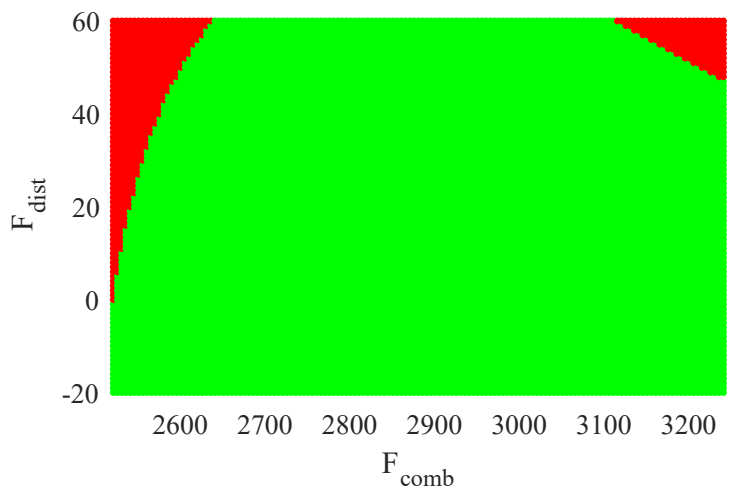

Figure 15: Jacobians that allow convergence at the reference value.

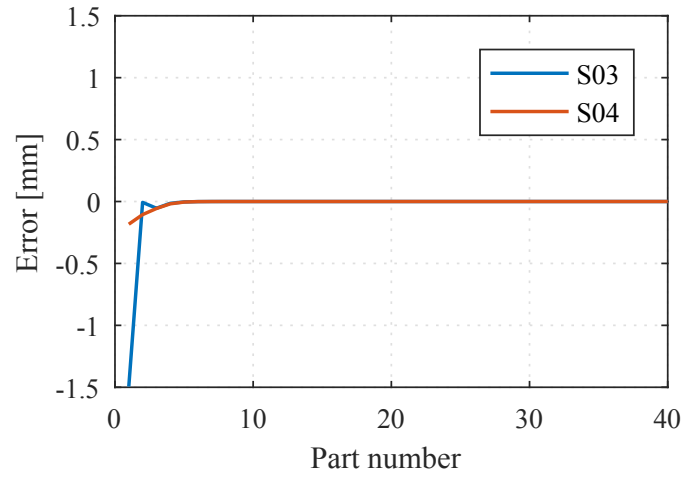

(a) Draw-in reaction

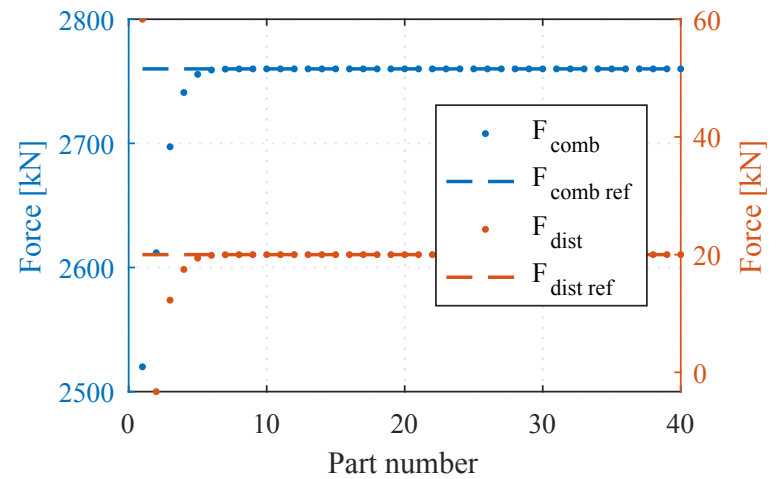

(b) Force reaction

Figure 16: Starting from red starting point $(2520 / 60)$

In addition to constraints due to quality parameters or actuator limits, in production it is useful to limit the actuator change per control cycle. For the case of the front fender, it can be useful to limit the force difference to $\Delta F_{\text {dist }}=20$ and the change in the accumulated force to $\Delta F_{c o m b}=100$. The results in figure 19 show a response that converges well, which also helps to stabilise the controller of the blank holder, as these sometimes need time to adjust to new values. The constrained control algorithm works for the fender and as expected, shows a similar response to the unbounded case, while there is an advantage in limiting the step size. Both controllers show a satisfactory behaviour for the front fender, while the strong non-linearity of the complex shape of the drawing part limits the controller gain significantly. The shown overshoots should be reduced or the step size limited before using the algorithm in a press shop. 


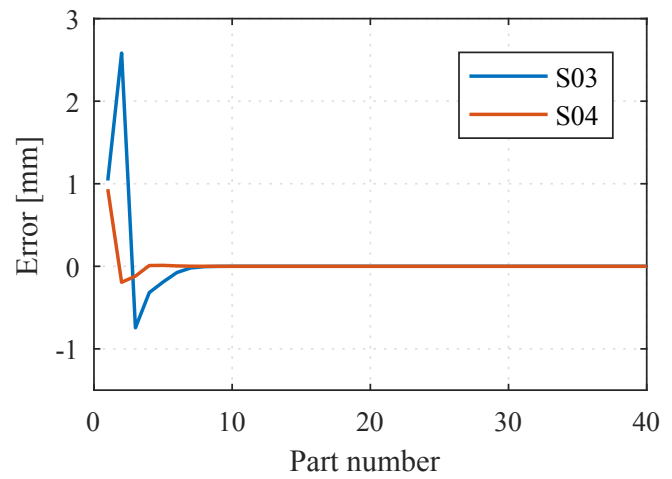

(a) Draw-in reaction

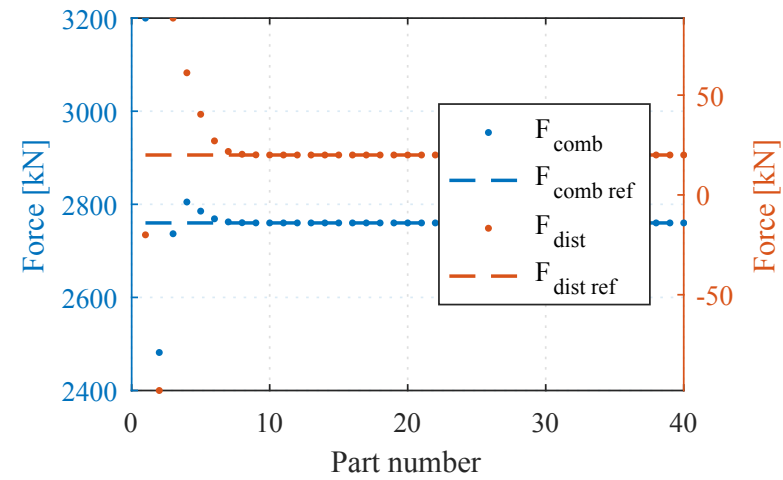

(b) Force reaction

Figure 17: Starting from green starting point (3200/-20)

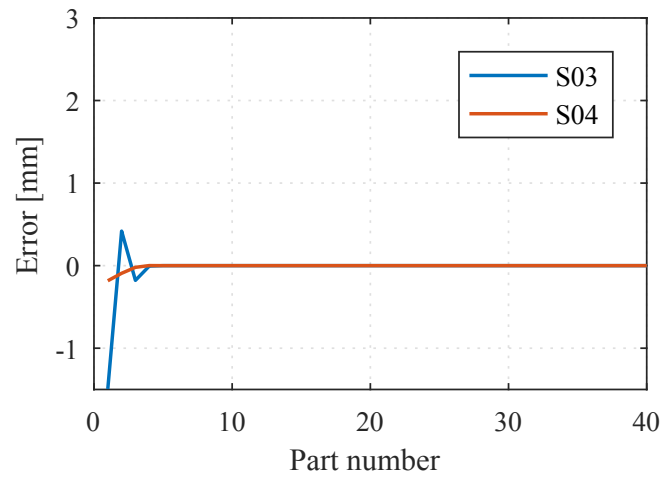

(a) Draw-in reaction

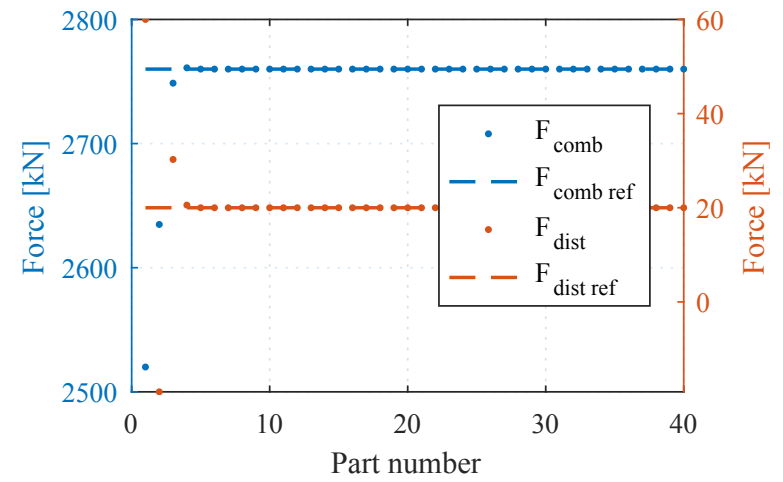

(b) Force reaction

Figure 18: Starting from green starting point (3200/-20) for bounded optimisation 


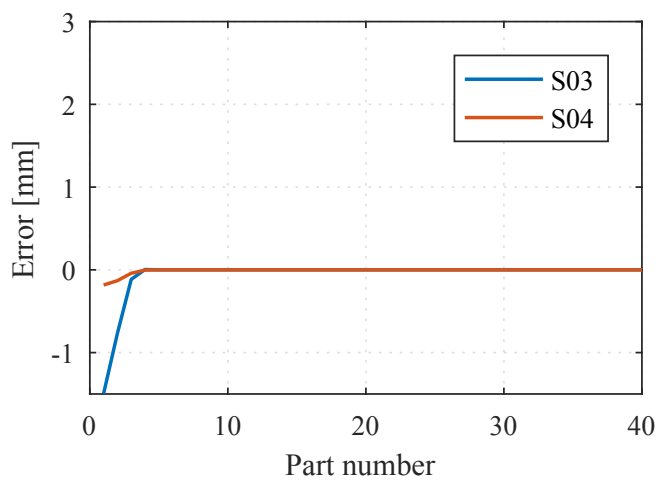

(a) Draw-in reaction

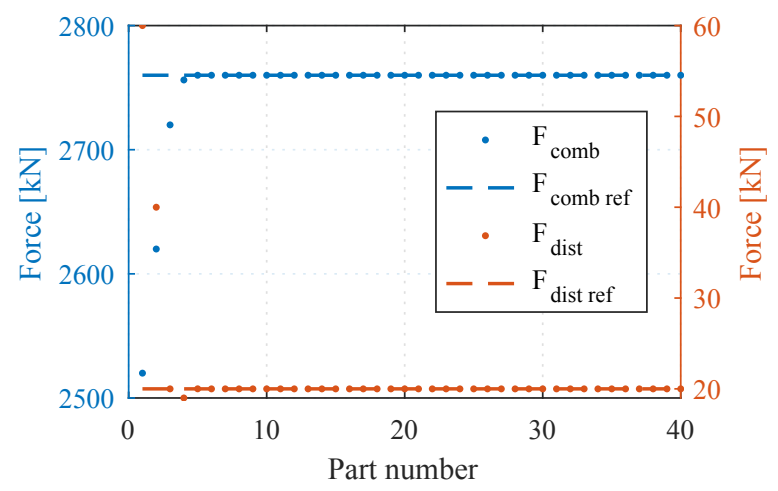

(b) Force reaction

Figure 19: Starting from green starting point (3200/-20) for bounded optimisation with step size limitation 


\section{Conclusion}

A comprehensive overview of the design of two different optimization approaches for part-to-part feedback control systems is given. Conditions on the controller gains that ensure the stability of the controller are derived for the unconstrained controller. Both algorithms show the ability to control the process for the non-linear process models by using a simple linearisation at the working point of the part. The kitchen sink shows a detailed approach for developing a controller based on a finite element model, while the front fender shows an experimental based approach.

The unconstrained optimization has the potential of a wide application especially, if the gain can be chosen sufficiently low to avoid overshoots. By using full models, the working range of the controller can also be determined, as shown for the kitchen sink. The constrained optimization on the other hand, provides the potential of avoiding infeasible situations through the introduction of constraints.

In general, both proposed algorithms have the potential to maintain the process at the nominal draw-in and as a result, improve the quality of the part in production.

\section{Acknowledgements}

The authors are grateful for the support of Daimler, as well as the CTI within the project 17366.1 PFIWIW. The authors also want to thank Franke, AutoForm and GOM for participating in the CTI project.

\section{References}

\section{References}

[1] D. Harsch, J. Heingärtner, Y. Renkci, P. Hora, Influence of scattering material properties on the robustness of deep drawing processes, in: 10th Forming Technology Forum. Model Based Control for Smart Forming Processes, 2017.

[2] R. Grueebler, P. Hora, Temperature dependent friction modeling for sheet metal forming, International Journal of Material Forming 2 (1) (2009) 251.

[3] D. Hortig, Experiences with the robustness of sheet metal forming processes, in: 4th Forming Technology Forum, 2011.

[4] K. Siegert, M. Ziegler, S. Wagner, Closed loop control of the friction force. deep drawing process, Journal of Materials Processing Technology 71 (1) (1997) 126 - 133.

[5] B. Griesbach, In-ProzeßStoffflußmessung zur Analyse und Führung von Tiefziehvorgängen, VDIVerlag, 2000.

[6] S.-W. Lo, T.-C. Yang, Closed-loop control of the blank holding force in sheet metal forming with a new embedded-type displacement sensor, The International Journal of Advanced Manufacturing Technology 24 (7) (2004) 553-559.

[7] C.-W. Hsu, A. Ulsoy, M. Demeri, Development of process control in sheet metal forming, Journal of Materials Processing Technology 127 (3) (2002) 361-368.

[8] B. Endelt, S. S. Tommerup, J. Danckert, A novel feedback control system - Controlling the material flow in deep drawing using distributed blank-holder force, Journal of Materials Processing Technology 213 (1) (2013) 36-50. 
[9] M. Barthau, Martin; Liewald, New Approach for Closed Loop Control of Deep Drawing Process for a Control Intervention during Deep Drawing Stroke, Advanced Materials Research 1140 (2016) 83-90.

[10] R. Mork, Qualitätsbewertung und Regelung für die Fertigung von Karosserieteilen in Presswerken auf Basis Neuronaler Netze, Ph.D. thesis, Technischen Universität München (2011).

[11] T. Bäume, W. Zorn, W.-G. Drossel, G. Rupp, Iterative process control and sensor evaluation for deep drawing tools with integrated piezoelectric actuators, Manufacturing Review 3 (2016) 3.

[12] B. Endelt, Proposing a new iterative learning control algorithm based on a non-linear least square formulation - Minimising draw-in errors, Journal of Physics: Conference Series 896 (2017) 012036.

[13] D. Harsch, J. Heingärtner, Y. Renkci, P. Hora, Influence of scattering material properties on the robustness of deep drawing processes, in: 10th Forming Technology Forum. Model Based Control for Smart Forming Processes, 2017.

[14] P. Fischer, D. Harsch, J. Heingärtner, Y. Renkci, P. Hora, A knowledge-based control system for the robust manufacturing of deep drawn parts, Procedia Engineering 207 (2017) 42-47.

[15] A. Neumann, Konzept zur Beherrschung der Prozessschwankungen im Presswerk, Ph.D. thesis, Bamberg (2014).

[16] M. Kraft, U. Bürgel, Novel concept for measurement of global blank draw-in when deep drawing outer skin automotive components, Journal of Physics: Conference Series 896 (2017) 012034.

[17] J. Heingärtner, P. Meyer-Galow, M. Gorji, P. Hora, Optical draw-in measurement for closed loop control of forming processes, in: Proceedings of IDDRG 2015, IDDRG 2015 Conference, 2015.

[18] F. M. Neuhauser, H. Mautz, N. Manopulo, M. Wahl, P. Hora, Observability of quality criteria in sheet metal parts during deep drawing operations using draw-in measurements, in: Proceedings of IDDRG 2017, 2017.

[19] M. Gu, S. C. Eisenstat, Efficient Algorithms for Computing a Strong Rank-Revealing QR Factorization, SIAM Journal on Scientific Computing 17 (4) (1996) 848-869.

[20] MATLAB, version 9.1.0.441655(R2016), The MathWorks Inc., Natick, Massachusetts, 2016.

[21] M. H. A. Bonte, Optimisation strategies for metal forming processes, Ph.D. thesis, Enschede (jun 2007).

[22] P. Fischer, J. Heingärtner, W. Aichholzer, D. Hortig, P. Hora, Feedback control in deep drawing based on experimental datasets, Journal of Physics: Conference Series 896 (2017) 012035.

[23] P. Fischer, D. Harsch, J. Heingärtner, Y. Renkci, P. Hora, Inline feedback control for deep drawing applications, IOP Conference Series: Materials Science and Engineering 159 (2016) 012006.

[24] M. Hans Christian Andreas Hänsel Deutschland, 1969 -, Nichtisothermes Werkstoffmodell für die FESimulation von Blechumformprozessen mit metastabilen austenitischen CrNi-Stählen, Ph.D. thesis, Düsseldorf (1998).

[25] F. Barlat, J. Brem, J. Yoon, K. Chung, R. Dick, D. Lege, F. Pourboghrat, S.-H. Choi, E. Chu, Plane stress yield function for aluminum alloy sheets-part 1: theory, International Journal of Plasticity 19 (9) (2003) 1297-1319. 